\title{
METHOD OF AIRBORNE SAR RADIATION CALIBRATION BASED ON POINT TARGET
}

\author{
YANG Shucheng ${ }^{1 *}$, XU. Zhida ${ }^{1,2}$, CHENG. Chunquan ${ }^{1}$ \\ ${ }^{1}$ Chinese Academy of Surveying and mapping, Beijing, China - (yangsc, cspring)@ casm.ac.cn \\ ${ }^{2}$ College of Geomatics, Shandong University of Science and Technologe, Qingdao, China - 15726234276@163.com
}

Commission I, WG I/4

KEY WORDS: Airborne SAR, Radiation Calibration, Antenna Pattern, Integral Method, Peak Method

\begin{abstract}
:
Radiation calibration of airborne SAR images is the basis for realizing the quantitative processing of airborne SAR images. Radiation calibration based on point targets is a general and convenient method. However, high precision is still the goal of improving the current point target SAR radiation calibration. For the key technology of high-precision radiation calibration processing for airborne SAR images, this paper uses the integral method and the peak method to calculate the point target radiation response energy, By analysing the radiation characteristics of the SAR image and comparing the methods, the sinc function model is used to fit the corrected antenna pattern. Combined with the radar power equation to solve the calibration constant, the complete point target radiation calibration method and process are established, and the different methods in the key technology are compared and analysed. The results show that the calibration method combined with the energy calculation model of the integral method and the sinc function antenna pattern correction model can achieve higher relative and absolute calibration accuracy. The optimized method can meet and exceed the requirements for radiometric calibration accuracy for high-resolution airborne SAR applications.
\end{abstract}

\section{INTRODUCTION}

With the development of new airborne SAR systems towards high resolution, multi-polarization and multi-imaging modes, the application of SAR is not limited to qualitative remote sensing using target position, texture, shape and other information for target discrimination. In some monitoring applications (such as water pollution monitoring, pest and disease monitoring, etc.), qualitative remote sensing will not meet the requirements of the application. This requires the quantitative back-scattering of ground features from SAR data, that is, quantitative remote sensing. Therefore, the process of obtaining the corresponding backscatter coefficient from the SAR image pixel intensity value is the radiation calibration. SAR radiation calibration is an important basis for the quantitative processing of SAR data.

Researchers in related fields at home and abroad have conducted a lot of research on SAR radiation calibration. At present, airborne SAR radiation calibration mainly uses point targets for calibration research. Point-based radiation calibration includes active point targets and passive point targets [7]. Passive point targets are radiated calibrations by deploying corner reflectors with specific dimensions and using their theoretical radar scattering cross-sectional area. The active point target can use the adjustment of the internal amplifier to obtain a larger radar scattering cross-sectional area, and can obtain better radiation calibration accuracy, but compared to the passive point target, its cost is higher and the processing is more complicated. Therefore, the current application is still more for the use of passive point targets for radiation calibration. Many experts and scholars have studied the radiation calibration of passive point targets. FENG Zongmin uses point targets to conduct radiation calibration studies on airborne SAR images and analyses the accuracy of radiation calibration [12]. Zheng Chen used the integration method and the peak method to perform airborne SAR radiation calibration experiments and perform accuracy analysis on point targets. The results show that the integration method is more stable than the peak method [11]. Ulander L M and others used point targets to conduct radiation calibration studies and evaluated the stability of the calibration results [5]. None of the above studies has studied and analysed the antenna pattern correction method, but only analysed the point target response energy calculation method. This paper focuses on the three key technical steps of calculating the response energy of point targets, calibrating antenna patterns, and calculating calibration constants for highresolution airborne SAR radiation calibration. In this paper, two point target response energy calculation methods are combined with the sinc function antenna pattern correction model to complete the radiation calibration. The two methods are evaluated and analysed using the combination of relative radiation calibration accuracy and absolute radiation calibration accuracy, so as to provide reference for high-resolution airborne SAR radiation calibration based on point targets.

\section{BASIC METHOD OF RADIATION CALIBRATION}

The SAR radiation calibration based on point targets is performed by setting a certain number of point targets with known scattering cross-sections in the calibration field, and calculating the correspondence between the target's backscatter coefficient and the SAR image intensity value according to the radar equation. For a stable SAR system, this correspondence is a calibration constant [11]. The calibration constant is used to calculate the ground backscattering coefficient to complete the

\footnotetext{
* Corresponding author
} 
radiation calibration. In the process of radiation calibration, three key steps are needed to calculate the response energy of the point target, correct the antenna pattern, and calculate the calibration constants. The SAR radiation calibration method flow is shown in Figure 1.

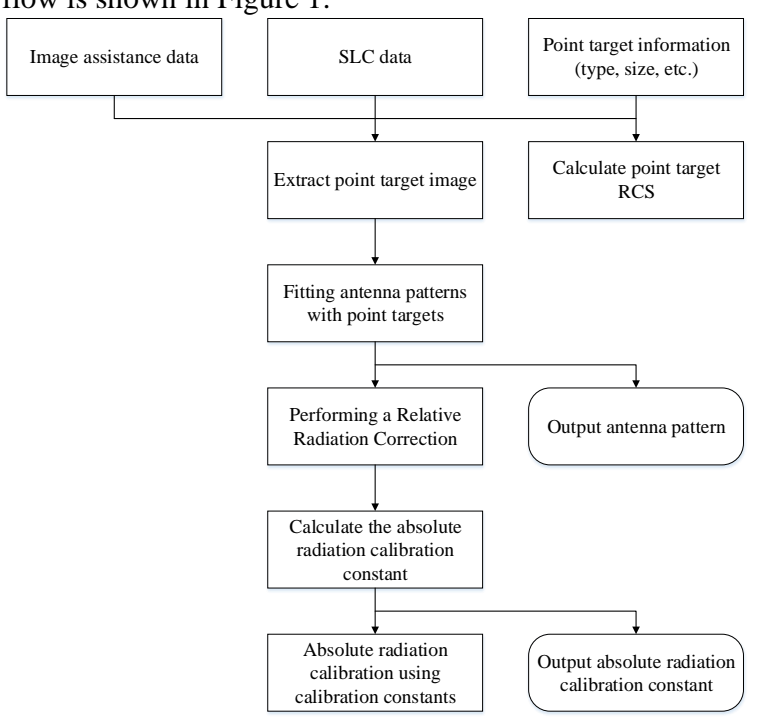

Figure.1 Point Target Radiation Calibration Flow Chart

\subsection{Point target response energy calculation}

When using point targets for radiation calibration, in order to be able to effectively extract point targets from the background, it is necessary to ensure that the point targets have a sufficient degree of visibility relative to the background. Therefore, the concept of signal-to-clutter ratio (SCR) was introduced to measure the visibility of point targets relative to the background [3], the calculation formula of SCR is:

$$
S C R=\frac{\sigma_{p q}^{T}}{\sigma_{p q}^{C}}=\frac{\sigma_{p q}^{T} \sin \theta_{i}}{\left\langle\sigma_{p q}^{C}\right\rangle p_{a} p_{r}}
$$

Where, $\sigma_{p q}^{T}$ is the radar cross section of the point target, $\left\langle\sigma_{p q}^{C}\right\rangle$ is the background average radar scattering crosssectional area. Previous studies have shown that when the signal-to-clutter ratio of a point target is greater than $20 \mathrm{~dB}$, the effect of background noise on the calibration result is less than $0.5 \mathrm{~dB}$ [4]. Therefore, whether the signal-to-clutter ratio is greater than $20 \mathrm{~dB}$ can be used as a verification condition for the validity of the point target.

Common methods for measuring the response energy of point targets include the peak method and the integral method. The peak method is to obtain the response energy of a point target by the peak response energy of the point target. In essence, the impulse response energy is obtained by calculating the product of the point target response peak value and the equivalent resolution unit area [11]. The peak method for calculating the impulse response energy of a point target is:

$$
\varepsilon_{r}=D N^{2} a b \delta_{a} \delta_{b}
$$

Where, $D N^{2}$ is the pixel intensity value of the peak point of the point target, $a$ and $b$ are the extensions of the antenna's $3 \mathrm{~dB}$ pulse response width in the azimuth and distance directions, $\delta_{a}$ and $\delta_{b}$ are the azimuth and distance pixel sizes.
The integration method is to obtain the response energy of the target point by integrating pixels within the range of the point target [8]. The response energy of a point target is equal to the total energy of the point target's response range on the image minus the background response energy. The response energy of a point target is equal to the total energy of the point target's response range on the image minus the background response energy. The specific formula is [11]:

$\varepsilon_{r}=\left(\sum_{i \in A}^{A} D N_{i}^{2}-\frac{N_{A}}{N_{B}} \sum_{i \in B}^{B} D N_{i}^{2}\right) \delta_{a} \delta_{b}$

In the formula, A is the point target integration area, $\mathrm{B}$ is the point target adjacent background area, $N_{A}$ is the number of pixels in the integration area, and $N_{B}$ is the number of pixels in the background area. Determine the integration window size based on the point target energy response area and the uniform background area. The integration window used in this paper based on the size of the response area of the point target in the image is shown in Figure 2. The gray filled area is the point target energy integration area, and the * symbol filled area is the background integration area.

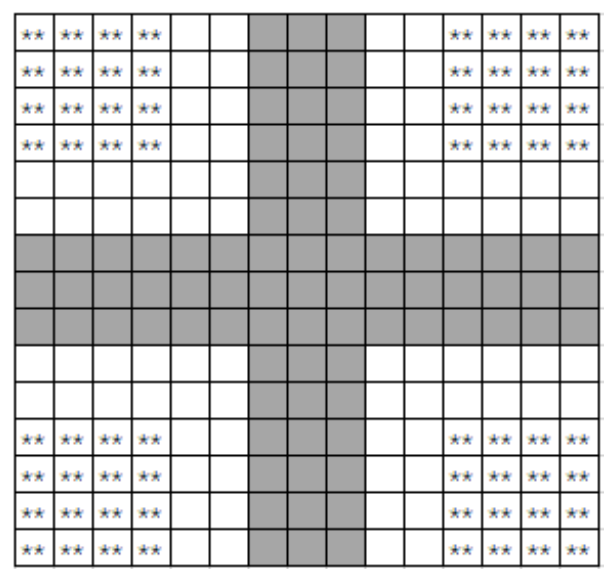

Figure.2 Integral Area Diagram

\subsection{Antenna pattern correction}

During the process of obtaining the SAR image, due to the change in the angle of the antenna pointing, there is a radiation deviation in the direction of the image. Therefore, before performing the absolute radiation calibration, the antenna pattern correction needs to be performed to complete the relative radiation correction. When determining the antenna pattern, the distance antenna pattern needs to be reconstructed according to the response energy of the point target, and the correction coefficient of each pixel is calculated by using the ratio of the distance direction maximum value and the distance direction fitted value obtained by the fitting function. The correction coefficient for each pixel using the obtained distance is used to correct the radiation deviation caused by the unknown antenna pattern. The uncorrected image appears bright in the middle and dark on both sides, and the overall brightness of the image after correction through the antenna pattern is relatively balanced [10].

Commonly used antenna pattern fitting functions include Chebyshev polynomial model, quadratic polynomial model and other fitting models [9]. This paper uses a quadratic polynomial 
model and a sinc function model to fit the antenna pattern and analyse the fitting results. The optimal fitting result is selected for antenna pattern correction. The fitting model of the sinc function is [1]:

$$
G=\chi_{1}\left(\sin c\left(\frac{\theta-\chi_{3}}{\chi_{2}}\right)\right)^{2}
$$

In the formula, $\chi_{1}$ is the amplitude coefficient, $\chi_{2}$ is the incident angle scale coefficient, $\chi_{3}$ is the offset of the incident angle on the horizontal axis, and $\theta$ is the size of the incident angle. In order to obtain an accurate estimate of $\chi_{1}, \chi_{2}, \chi_{3}$, an error equation is established to solve the best estimate of $\chi_{1}, \chi_{2}, \chi_{3}$ using a non-linear least square method. The error equation is:

$$
d_{G}=\min \sum_{i=1}^{N}\left(G\left(\chi_{1}, \chi_{2}, \chi_{3}, \theta_{i}\right)-G_{i}\right)^{2}
$$

In the formula, $\theta_{i}$ and $G_{i}$ are respectively the incident angle and the point target response energy value corresponding to the i-th point target. When ${ }_{G}$ gets the minimum value, get the best estimate of $\chi_{1}, \chi_{2}, \chi_{3}$, that is, get the antenna pattern model $\mathrm{G}$ [1]. The antenna pattern is used to calculate the distance correction coefficient for each pixel, and the radiation deviation caused by the unknown antenna pattern is corrected to complete the relative radiation calibration.

\subsection{Calculating the Calibration Constant}

After the antenna pattern is corrected, the absolute radiation calibration can be performed to obtain the radar backscattering coefficient. According to the radar imaging principle, the radar power received at a point on the ground is:

$$
P_{r}=\frac{P_{t} G_{\theta}^{2} \lambda^{2} \sigma_{\theta}}{(4 \pi)^{3} R^{4}}
$$

In the formula, $P_{r}$ is the received power, $P_{t}$ is the transmit power, $G^{\theta}$ is the antenna gain, $\lambda$ is the radar wavelength, $\sigma_{\theta}$ is the target scattering cross-sectional area, and $R$ is the distance from the radar antenna to the target. Within a synthetic aperture time $T_{a}$ ( $T_{a}$ is the accumulated time of the echo signal), and $F_{r}$ is the frequency of the SAR transmitting pulse, the number of transmitting pulses in a synthetic aperture time is $T_{a} F_{r}$. Therefore, the power of the SAR echo signal will increase a times, and the radar power accepted by the point target is [1]:

$$
P_{r}=\frac{P_{t} G_{\theta}^{2} \lambda^{2} \sigma_{\theta} T_{a} F_{r}}{(4 \pi)^{3} R^{4}}=C G_{\theta}^{2} \sigma_{\theta}
$$

Among them, let $C=\frac{P_{t} \lambda^{2} T_{a} F_{r}}{(4 \pi)^{3} R^{4}}$. That is, the overall relationship to determine the radiation calibration is:

$$
P_{r}=H \sigma_{\theta}
$$

This is the total transfer function of the SAR system.

In a SAR image, for any given pixel, the image amplitude value is proportional to the square of the power received [2],

$$
D N_{i}=k P_{i}^{1 / 2}
$$

According to the principle of the above formula, for a stable SAR system, the system transfer function is a constant $\mathrm{gg}$, and its calculation formula is [6]:

$$
K_{i}=\frac{E_{p i}}{\sigma_{r e f i}} \sin \theta
$$

In the formula, $K_{i}$ is the scaling constant corresponding to the point target i, $E_{p i}$ is the response energy corresponding to the point target, $\sigma_{\text {refi }}$ is the theoretical scattering cross-sectional area of the point target, and $\theta$ is the local incident angle corresponding to the point target.

Commonly used point targets include calibrators such as dihedral corner reflectors, trihedral corner reflectors, and conductive balls. Trihedral corner reflectors have a large radar backscatter cross-sectional area and are relatively stable. They are the most common corner reflectors used for radiation calibration. For a trihedral corner reflector, the theoretical scattering cross-sectional area is [8]:

$$
\sigma_{\text {refi }}=\frac{4 \pi a^{4}}{3 \lambda^{2}}
$$

In the formula, $\sigma_{\text {refi }}$ is the theoretical scattering crosssectional area of the corner reflector, $a$ is one side length of the trihedron, and $\lambda$ is the wavelength.

The overall image calibration constant is a calibration constant obtained by using $\mathrm{N}$ point targets. The overall image calibration constant is calculated as:

$$
K=\frac{1}{N} \sum_{i=1}^{N} K_{i}
$$

According to the principle described in the above formula, the calculation formula of the backscattering coefficient is [6]:

$$
\sigma^{0}=\frac{D N^{2}}{K} \sin \theta
$$

In the formula, $\sigma^{0}$ is the backscattering coefficient of the ground object, $D N^{2}$ is the corresponding SAR image intensity value, the image intensity value is the square of the amplitude value, $K$ is the calibration constant, and $\theta$ is the local incident angle corresponding to the point target. 


\section{INTRODUCTION OF TEST DATA}

In order to verify the calibration method described in this paper for the airborne SAR radiation calibration test, the calibration data used in the test is X-band airborne SAR data, and the data parameters are shown in Table 1 .

\begin{tabular}{|c|c|}
\hline parameter type & value \\
\hline radar wavelength & $0.031228 \mathrm{~m}$ \\
polarization mode & $\mathrm{HH}$ \\
Distance resolution & $0.187370 \mathrm{~m}$ \\
Azimuth resolution & $0.228278 \mathrm{~m}$ \\
Central angle of incidence & $43.97739^{\circ}$ \\
Aircraft altitude & $1016.928 \mathrm{~m}$ \\
\hline
\end{tabular}

Table.1 Test Data Parameter

According to the wavelength of the calibration data used, a trihedral corner reflector with a side length of 0.5 meters is used as the calibration point target. The calibration field is located in Danling City, Sichuan Province, China. Five corner reflectors are arranged along the distance in a uniform background area.

\section{TEST RESULTS AND ACCURACY ANALYSIS}

First measure the SCR value of each point target. If the SCR value of the point target is greater than $20 \mathrm{~dB}$, the point target is considered effective. The SCR measurement values of each point target are shown in Table 2. The SCR values of each point are greater than $20 \mathrm{~dB}$, and the average SCR value is $31.35 \mathrm{~dB}$, which indicates that the target data of each point meet the verification conditions.

\begin{tabular}{|c|c|c|c|}
\hline CR & $\begin{array}{c}\text { Image } \\
\text { coordinates } \\
(\mathrm{X})\end{array}$ & $\begin{array}{c}\text { Image } \\
\text { coordinates } \\
(\mathrm{Y})\end{array}$ & $\begin{array}{c}\text { SCR } \\
(\mathrm{dB})\end{array}$ \\
\hline 1 & 5765 & 2924 & 28.77 \\
2 & 7227 & 4439 & 28.93 \\
3 & 6854 & 5622 & 30.71 \\
4 & 6702 & 7342 & 33.36 \\
5 & 6651 & 9461 & 34.99 \\
\hline \multicolumn{2}{|c|}{ average RCS } & 31.35 \\
\hline
\end{tabular}

Table.2 SCR Statistics Table Corresponding to Different Point Targets

After extracting the point target according to the abovementioned radiation calibration principle, the point target is used to fit the antenna pattern to correct the antenna pattern to complete the relative radiation calibration. The point target response energy calculated using the integral method, the quadratic polynomial and the sinc function model were used to fit the antenna pattern of the experimental data, and the fitting results are shown in Figure 3 and Figure 4. It can be clearly observed from Figure 3 that the quadratic polynomial fitting antenna pattern has negative values at both ends of the image, which is inconsistent with the physical characteristics of the antenna pattern. The fitting of the antenna pattern based on the sinc function is consistent with the physical characteristics of the antenna pattern. Table 3 shows the comparison between the fitted value of the sinc function and the original value. Therefore, using the antenna pattern fitting result of the sinc function to correct the antenna pattern of the SAR image, the relative radiation calibration is completed. When the antenna pattern is not corrected, the entire SAR image appears bright in the middle and dark in both sides due to the radiation deviation. After the antenna pattern is corrected, the overall brightness of the image is more balanced. The corrected images of the antenna pattern are shown in Figure 5.

\begin{tabular}{|c|c|c|c|}
\hline CR & $\begin{array}{c}\text { CR energy } \\
\text { value }\end{array}$ & $\begin{array}{c}\text { fitted } \\
\text { value }\end{array}$ & ratio \\
\hline 1 & 1028870 & 1017962 & 0.99 \\
2 & 1360339 & 1362270 & 1.00 \\
3 & 1283806 & 1293707 & 1.01 \\
4 & 952057 & 911374 & 0.96 \\
5 & 414283 & 415722 & 1.00 \\
\hline
\end{tabular}

Table.3 Sinc Function Model Fitting Result Table

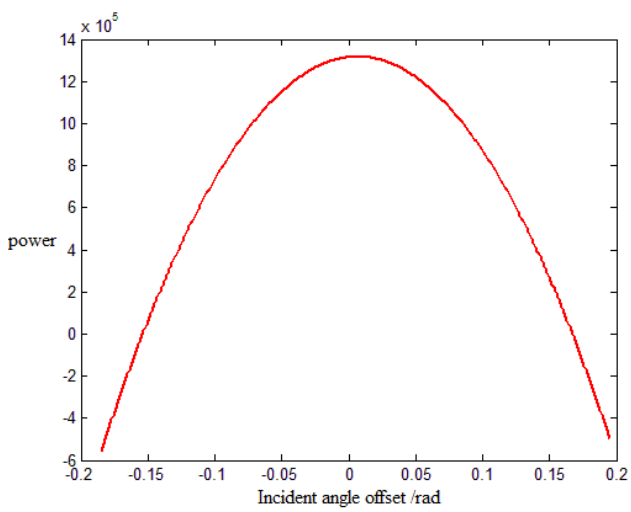

Figure.3 Quadratic Polynomial Antenna Pattern Fitting Graph

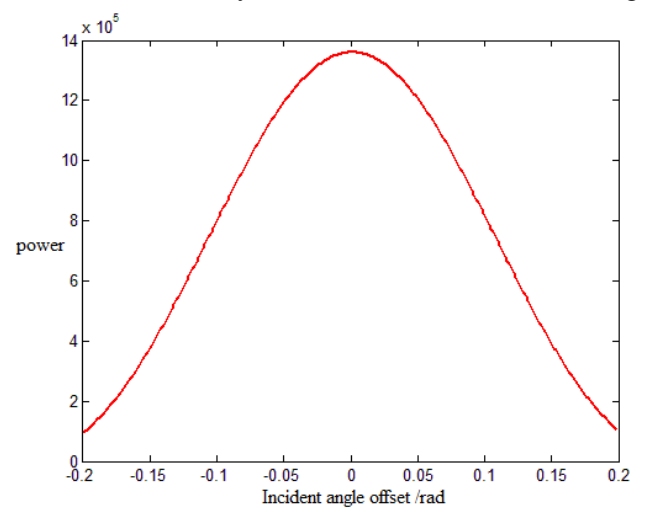

Figure.4 Sinc Function Antenna Pattern Fitting Graph

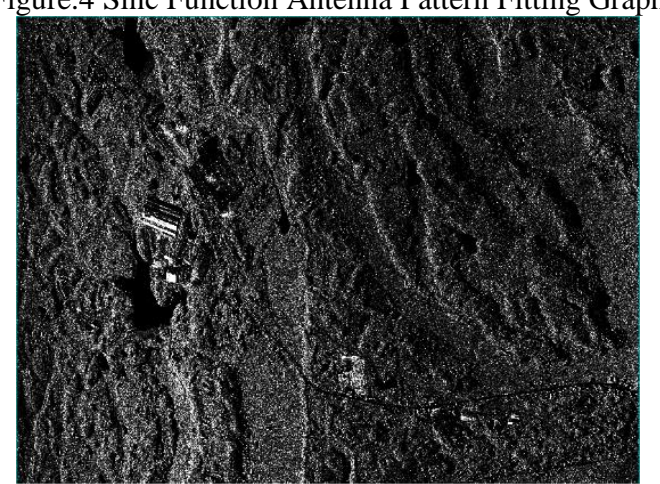

Figure.5 Antenna pattern corrected image

According to the radiation calibration principle, the above two point target response energy calculation methods are used to obtain the response energy of each point target after correction of the antenna pattern and calculate its calibration constant. In theory, the calibration constants corresponding to each point target should be equal, so the relative radiation calibration accuracy of the integration method and the peak method can be verified by verifying the standard deviation of the calibration constant calculated by each point target. The test results show that the relative radiation calibration accuracy of the integration method is $0.42 \mathrm{~dB}$, and the relative radiation calibration 
accuracy of the peak method is $0.50 \mathrm{~dB}$. According to the relative calibration results, the relative radiation calibration accuracy based on the integration method is better than that of the peak method, which indicates that the integration method has better stability than the peak method and the relative calibration results are more accurate. The relative calibration results of the two methods are shown in Table 4 and Table 5.

\begin{tabular}{|c|c|c|}
\hline CR & CR energy $(\mathrm{dB})$ & calibration constant $(\mathrm{dB})$ \\
\hline 1 & 61.41 & 34.95 \\
2 & 61.35 & 35.25 \\
3 & 61.33 & 35.44 \\
4 & 61.55 & 35.90 \\
5 & 61.35 & 35.91 \\
\hline \multicolumn{2}{|c|}{ Average calibration constant } & 35.51 \\
\hline \multicolumn{2}{|c|}{ Calibration constant standard } \\
deviation
\end{tabular}

Table.4 Integral Method Calibration Constant Statistics Table

\begin{tabular}{|c|c|c|}
\hline CR & CR energy $(\mathrm{dB})$ & calibration constant $(\mathrm{dB})$ \\
\hline 1 & 56.62 & 30.15 \\
2 & 56.71 & 30.61 \\
3 & 56.84 & 30.95 \\
4 & 56.36 & 30.71 \\
5 & 56.96 & 31.53 \\
\hline \multicolumn{2}{|c|}{ Average calibration constant } & 30.81 \\
\hline \multicolumn{2}{|c|}{ Calibration constant standard } \\
deviation
\end{tabular}

Table.5 Peak Method Calibration Constant Statistics Table

According to the principle of radiation calibration, the average value of all calibration constants is taken as the overall image calibration constant, and absolute radiation calibration is performed on the SAR image. Absolute radiation calibration of an existing point target to obtain its backscattered crosssectional area. The difference between the backscattered crosssectional area of the point target after calibration and the theoretical backscattered cross-sectional area is taken as the absolute value to determine the accuracy of the absolute radiation calibration. The absolute radiation calibration accuracy results are shown in Table 6 and Table 7 . The absolute maximum value of the absolute value of the difference between the RCS measurement value and the theoretical value of the point target after the absolute radiation calibration is taken as the absolute radiation calibration accuracy. According to Table 6 and Table 7, the absolute radiation calibration accuracy of the integration method is $0.56 \mathrm{~dB}$, and the absolute radiation calibration accuracy of the peak method is $0.72 \mathrm{~dB}$. It can be seen that the absolute radiation calibration accuracy of the integration method is better than the peak method.

\begin{tabular}{|c|c|c|c|c|}
\hline CR & $\begin{array}{c}\text { CR } \\
\text { thearetical } \\
\text { RCS/dB }\end{array}$ & $\begin{array}{c}\text { after } \\
\text { calibration } \\
\text { RCS/dB }\end{array}$ & $\begin{array}{c}\text { difference } \\
/ \mathrm{dB}\end{array}$ & $\begin{array}{c}\text { calibration } \\
\text { accuracy/dB }\end{array}$ \\
\hline 1 & 24.29 & 23.73 & 0.56 & \\
2 & 24.29 & 24.03 & 0.26 & 0.56 \\
3 & 24.29 & 24.22 & 0.07 & \\
4 & 24.29 & 24.68 & 0.39 & \\
5 & 24.29 & 24.69 & 0.40 & \\
\hline
\end{tabular}

Table.6 Integral method Absolute Calibration Accuracy Verification Table

\begin{tabular}{|c|c|c|c|c|}
\hline CR & $\begin{array}{c}\text { CR } \\
\text { thearetical } \\
\text { RCS/dB }\end{array}$ & $\begin{array}{c}\text { after } \\
\text { calibration } \\
\text { RCS/dB }\end{array}$ & $\begin{array}{c}\text { difference } \\
/ \mathrm{dB}\end{array}$ & $\begin{array}{c}\text { calibration } \\
\text { accuracy/dB }\end{array}$ \\
\hline 1 & 24.29 & 23.63 & 0.66 & \\
2 & 24.29 & 24.08 & 0.21 & \\
3 & 24.29 & 24.42 & 0.13 & 0.72 \\
4 & 24.29 & 24.18 & 0.11 & \\
5 & 24.29 & 25.01 & 0.72 & \\
\hline
\end{tabular}

Table.6 Peak method Absolute Calibration Accuracy Verification Table

Different applications of SAR imagery have different requirements for radiation calibration accuracy, including absolute radiation calibration accuracy and relative radiation calibration accuracy. Based on the requirements for radiation calibration accuracy of various applications, the relative radiation calibration accuracy of radiation calibration should be less than $0.5 \mathrm{~dB}$, and the absolute radiation calibration accuracy should be less than $1.0 \mathrm{~dB}$ [3]. In this experiment, the relative calibration accuracy of the integral method is $0.42 \mathrm{~dB}$ and the absolute calibration accuracy is $0.56 \mathrm{~dB}$. The relative calibration accuracy of the peak method is $0.50 \mathrm{~dB}$ and the absolute calibration accuracy is $0.72 \mathrm{~dB}$. Both methods can meet the calibration accuracy requirements. However, the integration method is superior to the peak method in both relative calibration accuracy and absolute calibration accuracy, indicating that the integration method is superior to the peak method in terms of calibration stability and accuracy. In addition, according to the theoretical analysis, it is known that when the peak target method is used to calculate the response energy of a point target, if high accuracy is required, the system must be stable and the imaging quality is good. At the same time, the point target used should have a large scattering crosssectional area. For the application of airborne SAR radiation calibration, the stability of the system is poorer than that of the spaceborne SAR system due to the impact of wind and selfvibration on the platform of the airborne SAR, making the peak method applicable conditions not well guaranteed. However, point targets are generally arranged in a uniform background area to ensure the premise of using the integration method. Therefore, using the integration method is easier to obtain more stable and accurate calibration results when the image imaging quality is unknown. According to the theoretical analysis and experimental results in this paper, the radiation calibration strategy using the integral method to calculate the response energy of the point target and the antenna pattern correction using the sinc function model is more suitable for highresolution airborne SAR radiation calibration applications.

\section{CONCLUSION}

SAR radiation calibration is a key step for SAR images from qualitative applications to quantitative applications, which can greatly expand the application scope and application capabilities of SAR images. This paper uses X-band highresolution airborne SAR images to perform radiation calibration experiments based on the passive point target method. The integral method and the peak method are used to obtain the response energy of the point target. The sinc function model is used to fit the antenna pattern to perform relative radiation correction, and then the image calibration constant is calculated to perform absolute radiation calibration on the image. According to the test results, the calibration results of the two methods are analysed by using a combination of relative calibration accuracy and absolute calibration accuracy. The results show that the integration method is better than the peak 
method in stability, indicating that the radiation calibration strategy that uses the integration method to calculate the point target response energy and use the sinc function model to fit the antenna pattern is more suitable for high-resolution airborne $\mathrm{SAR}$ radiation calibration applications.

\section{ACKNOWLEDGEMENTS}

This work was supported by the National Key Research and Development Program of China, No. 2018YFF0215303 and 2017 YFB0503004.

\section{REFERENCES}

CHEN Jinxing, ZHANG Bo, WANG Chao, et al. Comparetive Study on the Application of Two Corner Reflector Based Radiometric Calibration Methods for High Resolution Airborne SAR Image[J]. REMORE SENSING TECHNOLOGY AND APPLICATION, 2015,30(4):677-683.

Dobson M C,Ulaby F T,Brunfeldt D R,etal.External Calibration of Sir-B Imagery with Area extended and Point Targets[J].IEEE Transactions on Geoscience and Remote Sensing, 1986, 24(4):453-461.

FREEMAN A. SAR calibration:An overview[J].IEEE Transaction on Geoscience and Remote Sensing, 1992, 30(6):1107-1121.

Touzi R, Hawkins R K, Cote S. High-Precision Assessment and Calibration of Polarimetric RADARSAT-2 SAR Using Transponder Measurements[J]. IEEE Transactions on Geoscience \& Remote Sensing, 2013, 51(1):487-503.

Ulander L M H.Accuracy of using point targrts for SAR calibration[J].IEEE Transaction on Geoscience and Remote Sensing,1991,27(1):139-148.

WANG Ji, LI Song, WENG Yinkan, et al. A Model of Geometric Correction and Radiation Calibration for TrrraSARX Image[J]. Science Technology and Engineering, 2017,17(14): 223-227.

YUAN Lihai, GE Jialong, JIANG Kai et al. Analysis and Design of SAR Radiometric Calibration Precision[J]. Radar Science and Technology,2009,2(1):35-39.

YUAN Lihai, LI Zhao, GE Jialong, et al. Research on Approach of SAR Radiometric Calibration Using Point Target[J]. RADIO ENGINEERING, 2009, 39(1):25-28.

ZHANG Ting. Study on Airborne SAR Calibration Technology[D]. Beijing: Peking University, 2012.

ZHENG Chen. Hige Resolution Airborne SAR Calibration Technology Research and Precision

ZHENG Chen, HUANG Lei, CHEN Quan. Accuracy of Airborne SAR Radiometric Calibration With Point Target[J]. Remote Sensing Information, 2015,30(4):14-19.

Zongmin F, Lei H, Zhihua T, et al. Airborne SAR radiometric calibration using point targets[C]. 35th International Symposium on Remote Sensing of Environment 2014. 